\title{
In situ TEM Observation of Lithiation and Sodiation Process of $\mathrm{ZnO}$ Nanowire
}

Hasti Asayesh-Ardakani ${ }^{1,3}$, Anmin Nie ${ }^{1,3}$, Farzad Mashayek ${ }^{2}$, Robert F. Klie ${ }^{3}$ and Reza Shahbazian-Yassar ${ }^{1,2,3}$

${ }^{1}$ Department of Mechanical Engineering-Engineering Mechanics, Michigan Technological University, Houghton, MI 49933-1295, USA

${ }^{2}$ Department of Mechanical and Industrial Engineering, University of Illinois at Chicago, Chicago, IL60607-7059, USA

${ }^{3}$ Department of Physics, University of Illinois at Chicago, Chicago, IL60607-7059, USA

The limited source of $\mathrm{Li}$ and over-growing demands of high capacity storage for application in electrical vehicles and green power backup energy makes the Li-ion battery an expensive choice for future. This need triggers the new battery concept search beyond Li-ion. Natural abounding of $\mathrm{Na}$ on earth makes it more affordable and eco-friendly choice. Another considerable issues in batteries are performance and cyclability of batteries. The anode materials usually experience large volume changes through the ion insertion and extraction. This volume change and lithium embrittlement usually causes cracks and loss of contact in the anode material, which ultimately causes the failure of battery.

In this work, we investigated and compared the structural and mechanical changes of $\mathrm{ZnO}$ nanowires during sodiation and lithiation process by using aberration corrected scanning transition electron microscopy and in situ TEM (Figure 1 shows a pristine ZnO nanowire and corresponding SAED pattern before and after lithiation). The cracks were created upon the first lithiation process of single crystalline $\mathrm{ZnO}$ nanowire. The lithiated $\mathrm{ZnO}$ nanowire shows multiple glassy domains, which has low strength and ductility. This results in poor cyclability of battery. On the other hand, $\mathrm{ZnO}$ nanowire after sodiation show dislocations on the surface of nanowire that results in more ductility of sodiated nanowire rather than lithiated one. Another important issue is the control of reaction rate. In the fast reaction rate, the large Zn nanocrystals can grow and suppress the formation of $\mathrm{Zn}$ alloys and dramatically reduce the capacity of battery. This direct comparison demonstrates the critical role of anode material's mechanical properties and reaction rates on failure mechanism and cyclability of Li/Na-ion batteries. 

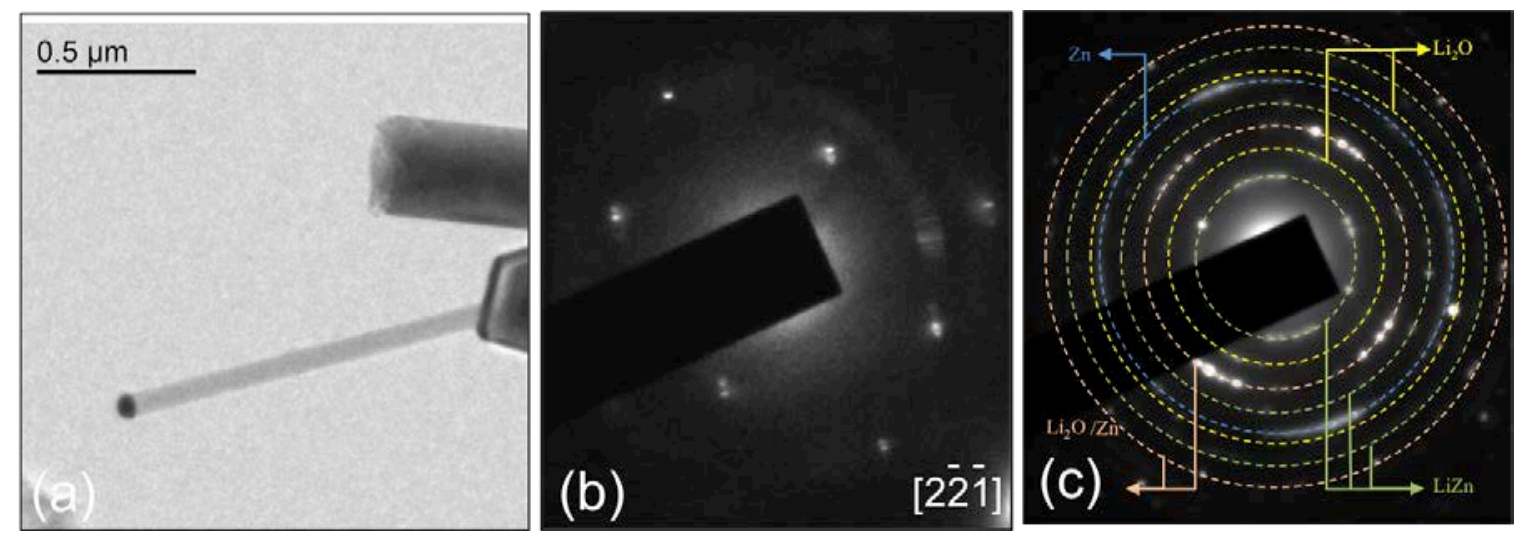

Figure 1. In situ lithiation of single crystalline $\mathrm{ZnO}$ nanowire. (a) pristine $\mathrm{ZnO}$ nanowire and (b) selected area electron diffraction pattern of pristine nanowire. (c) The selected area diffraction pattern of lithiated nanowire 